\title{
Cold streams: detectability, relation to structure and characteristics
}

\author{
Tobias Goerdt \\ Department for Astrophysics, University of Vienna, \\ Türkenschanzstr. 17, 1180 Vienna, Austria \\ email: tobias.goerdt@univie.ac.at
}

\begin{abstract}
Cold gas streaming along the dark-matter filaments of the cosmic web is predicted to be the major provider of resources for disc buildup and star formation in massive galaxies in the early universe. We use hydrodynamical simulations to study to what extent these cold streams are traceable in the extended circum-galactic environment of galaxies via Ly alpha emission, Ly alpha absorption and selected low ionisation metal absorption lines. We predict the strength of the absorption signal produced by the streams and find that it is consistent with observations in high redshift galaxies. The characteristics of the Ly alpha emission of our simulated galaxies are similar in luminosity, morphology and extent to the observed Ly alpha blobs, with distinct kinematic features. We analyse the characteristics of the cold streams in simulations and present scaling relations for the amount of infall, its velocity, distribution and its clumpiness and compare our findings with observations.
\end{abstract}

Keywords. cosmology: theory - galaxies: evolution - galaxies: formation - galaxies: high redshift - intergalactic medium — galaxies: ISM

\section{Introduction}

Our understanding of how galaxies form has changed substantially in recent years. A decade ago it was thought that galaxies collect their baryons through diffuse gas, spherically symmetrically falling into dark matter haloes and being shock-heated as it hits the gas residing in the halos, the so-called hot mode accretion. Whether the gas eventually settles into the equatorial plane, forming a galactic disk was depending on the mass of the dark halo. Below a critical mass, the gas could cool efficiently, forming a disk galaxy, while for larger masses the cooling time would be longer than the Hubble time, leading to structures that resemble galaxy clusters with a large baryon fraction in the hot, diffuse intergalactic gas component. Recent theoretical work and simulations (Dekel et al. 2009) however have shown that at high redshift $(z \gtrsim 2)$, galaxies acquire their baryons primarily via cold streams of relatively dense and pristine gas with temperatures around $10^{4} \mathrm{~K}$ that penetrate through the diffuse shock-heated medium, the so-called cold mode accretion. These streams peak in activity around redshift 3 .

$N$-body simulations suggest that about half the mass in dark-matter haloes is built-up smoothly, suggesting that the baryons are also accreted semi-continuously as the galaxies grow. Hydrodynamical cosmological simulations also show rather smooth gas accretion, including mini-minor mergers with mass ratios smaller than 1:10, that brings in about two thirds of the mass (Dekel et al. 2009). The massive, clumpy and star-forming discs observed at $z \sim 2$ may have been formed primarily via the smooth and steady accretion provided by the cold streams, with a smaller contribution by major merger events. 


\section{Absorption line profiles}

In this section $\dagger$ we consider the Ly $\alpha$ and metal line absorption that occurs as UV light emitted by the central galaxy is absorbed by gas in the circum-galactic environment. This is defined to be situated in the spherical zone from just outside the galactic disk to around the virial radius $R_{\mathrm{v}}$. Observations of absorption against the central galaxy itself have the advantage of being able to discriminate between inflows and outflows because the absorption may be assumed to occur in foreground material only. Radiation emitted or scattered from behind the galaxy is blocked by the galaxy itself. However, such absorption do not provide spatial information about the distance from the galaxy centre as they are all by definition at an impact parameter $b=0$ from the galaxy centre.

Along a given sight-line the gas has a varying density, temperature and radial velocity as function of radial position $r$ from the central galaxy. The convolutions of the different densities and radial velocities in the gas along the line of sight are the main ingredients to compute an absorption line profile. For a fair comparison to the observations, we mimic a Gaussian point spread function. It has a beam-size (= FWHM $\eta$ of the Gaussian) of $4 \mathrm{kpc}$.

The likelihood of a detection of a cold stream while looking at a single galaxy from a single direction without averaging is for $\mathrm{Mg}$ II: an inflow $>150 \mathrm{~km} \mathrm{~s}^{-1}$ with an EW $>0.2 \AA$ in $1.3 \%$ of all observations. These values should be achievable by future observations. We produce stacked spectra using our simulations by summing up the line profiles of several thousand different directions for each of the three galaxies and stack them together. We determine the absorption line profile for a spherical shell between an outer radius and an inner radius. The predicted metal line absorption profiles appear tiny. Probably the most suitable lines for the purpose of detecting cold streams in absorption are C II and Mg II since they have the strongest signal. Out of those Mg II is closer to being observed with the needed sensitivity and resolution.

\section{Cold streams as Ly $\alpha$ blobs}

Hydrodynamical cosmological simulations robustly demonstrate that massive galaxies at high redshifts were fed by cold gas streams, inflowing into dark-matter haloes at high rates along the cosmic web. In this sectionf we show that these streams should be observable as luminous $\mathrm{L} \alpha$ sources, with elongated irregular structures stretching for distances of over $100 \mathrm{kpc}$. The release of gravitational potential energy by the instreaming gas as it falls into the halo potential well is the origin of the $\mathrm{L} \alpha$ luminosity. The predicted $\mathrm{L} \alpha$ emission morphologies and luminosities make such streams likely candidates for the sources of observed high redshift $\mathrm{L} \alpha$ blobs. Most of the gas in the cold streams is at temperatures in the range $(1-5) \times 10^{4} \mathrm{~K}$, for which the $\mathrm{L} \alpha$ emissivity is maximised.

Using state-of-the-art cosmological AMR simulations with 70 pc resolution and cooling to below $10^{4} \mathrm{~K}$, and applying a straightforward analysis of $\mathrm{L} \alpha$ emissivity due to electron impact excitation, we produced maps of $\mathrm{L} \alpha$ emission from simulated massive galaxies at $z \sim 3$. We computed the average $L \alpha$ luminosity per given halo mass and the predicted luminosity function of these extended $\mathrm{L} \alpha$ sources, with an uncertainty of \pm 0.4 dex. The properties of the individual images resemble those of the observed LABs in terms of morphology and kinematics. The predicted luminosity function is close to the observed LAB luminosity function. The simulated LABs qualitatively reproduce the 
observed correlations between the global LAB properties of $\mathrm{L} \alpha$ luminosity, isophotal area and linewidth.

\section{Relation to structure}

We know that gas and, for our purposes more interestingly, dark matter haloes hosting satellite galaxies enter a host halo via smooth accretion streams (Dekel et al. 2009). Suppose now that galaxies and their satellite systems form in the focal point of a number $m$ of randomly oriented cold streams. What is then the probability that a certain fraction of satellites lies within a thin plane around the central galaxy of the host halo? 15 out of Andromeda galaxy's 27 satellites are within a plane with $200 \mathrm{kpc}$ radius and $12.6 \mathrm{kpc}$ vertical rms scatter. In order to work out the likelihood to have of order $56 \%$ of satellites in a plane as thin as 3.8 degree we have performed Monte Carlo experiments, drawing randomly orientated streams and calculating the probabilities of having a given ratio of subhaloes within a plane of certain thickness $\dagger$.

Since we assumed that all the streams pass trough the galaxy centre, two streams will always lie on a perfect plane. The ' $2 / m$ ' values therefore are always at probability $P=1.0$. For a three stream scenario we would expect at least $67 \%$ of the satellites to lie within one plane. Since two, three or four stream configurations are most commonly seen in cosmological simulations, we can already say that there is nothing surprising about the configuration seen around the Andromeda galaxy. On the contrary, the fact that so little satellites lie on a thin plane rules out a two stream or a three stream scenario for the Andromeda galaxy, unless secular evolution or other processes move some of the satellites out of that plane. Turning the argument around, the fact that we observe a large fraction of satellites in a thin plane can be seen as indirect observational evidence for the cold mode accretion stream scenario proposed by Dekel et al. (2009) and that scattering events are not efficient, at least for the Local Group.

\section{Streaming rates into high- $z$ galaxies: smooth flows and mergers}

In this section we look at the inflow rates of cold accretion streams, at their average inflow velocities, at the distribution of inflow rates as well as the role of mergers versus smooth flows. For this purpose we analyse the clumpiness of the gas streams and use the sharp peaks of inflow in the $\dot{M}(r)$ profiles as well as a new and unique clump finder. We evaluate each clump mass and estimate a mass ratio for the expected merger, ignoring further mass loss in the clump on its way in. We find: (1) The velocity profiles for the cold streams are by and large constant and show no sign of free-fall at all. (2) The constant infall velocity follows power law relations with respect to the halo mass and to the redshift. (3) The inflow distribution can be described by a double-Gaussian distribution (i.e. the sum of two Gaussians). (4) Most of the amount of inflow ( $>75 \%$ ) is entering the halo at low inflow rates (i.e. smooth accretion) and only a small portion $(<25 \%)$ of the inflow is coming in at high inflow rates (massive merger events). (5) Merger rates follow for a given mass and redshift bin of galaxies a robust double-logarithmic behaviour (i.e. the convolution of two logarithmic functions).

Interestingly, observer also found a double-Gaussian distribution when looking at the specific star formation rate distribution at fixed stellar mass in observations. They interpreted this as contributions from main-sequence and starburst activity. The analogy between their and our findings indicates that connections might exist between smooth

$\dagger$ published as Goerdt \& Burkert (2013) 
accretion and main-sequence star formation on the one hand as well as accretion through merger events and starburst activity star formation on the other side.

\section{Conclusions}

We conclude that the signatures of cold inflows are subtle, and when stacked are overwhelmed by the outflow signatures. Our predicted Ly $\alpha$ line absorption profiles agree with the observations, while the stacked metal line absorption from the inflows is much weaker than observed in the outflows. The single-galaxy line profiles predicted here will serve to compare to single-galaxy observations.

Our results support the idea that the observed LABs are direct detections of the cold steams that drive the evolution of massive galaxies at high redshifts. Even though the observed LABs are sometimes associated with central sources that are energetic enough to power the observed L $\alpha$ emission, such as starbursts and AGNs, these central sources are very different from each other in the different galaxies, and in many LABs they are absent altogether. The gravitational heating associated with the inflowing cold streams is a natural mechanism for driving the extended $\mathrm{L} \alpha$ cooling radiation observed as LABs, and this extended $\mathrm{L} \alpha$ emission is inevitable in most high-redshift galaxies.

We conclude that the special spatial alignment of Andromeda's satellite galaxies can naturally be explained by cold stream accretion and simple geometry. Hydrodynamical simulations naturally produce coplanar structures of satellites via cold mode accretion streams. Satellite alignments around galaxies can be seen as indirect observational evidence for the cold stream paradigm and provides important information about the accretion history of galaxies.

We conclude that gas is flowing into a galaxy's halo mainly as smooth accretion flows with only a minority $(\sim 25 \%)$ coming in via merger events such as clumps or small satellite galaxies. The velocity profile of those streams is, contrary to what might be expected, constant and not free-falling. The potential energy of the gas which is lost on its way in must be dissipated by other mechanisms, such as Ly $\alpha$ radiation (Goerdt et al. 2010). The analogousness of the equations used to describe the distribution of the gas inflow in simulations or to describe the specific star formation rate distribution in observations (the double-Gaussian decomposition) indicates a connection might exist between smooth accretion and main-sequence star formation on the one hand side as well as a connection between accretion through merger events and starburst activity star formation on the other hand.

\section{Acknowledgements}

Tobias Goerdt is a Lise Meitner fellow. This work was supported by FWF project number M 1590-N27, the Spanish Ministerio de Economía y Competitividad (MINECO) via the projects AYA2009-13875-C03-02 and AYA2012-31101 and via the JdC subprogramme JCI-2011-10289.

\section{References}

Dekel, A. et al. 2009, Nature, 457, 451

Goerdt, T., Dekel, A., Sternberg, A., Ceverino, D., Teyssier, R., \& Primack, J. R., 2010, MNRAS, 407,613

Goerdt, T., Dekel, A., Sternberg, A., Gnat, O., \& Ceverino, D., 2012, MNRAS, 424, 2292

Goerdt, T. \& Burkert, A., 2013, arXiv:1307.2102 